\title{
Face validity of a synthetic papilla designed for biliary sphincterotomy training $\square$
}

\section{다)(우우}

\section{Authors}

Sophia E. van der Wiel, Arjun D. Koch, Marco J. Bruno

\section{Institution}

Department of Gastroenterology and Hepatology, Erasmus MC, University Medical Center Rotterdam, The Netherlands

submitted 19.9.2018

accepted after revision $\quad 27.12 .2018$

Bibliography

DOI https://doi.org/10.1055/a-0842-6369 |

Endoscopy International Open 2019; 07: E757-E761

(c) Georg Thieme Verlag KG Stuttgart · New York elSSN 2196-9736

Corresponding author

Arjun D. Koch, MD, PhD, Erasmus MC, University Medical Center Rotterdam, Department of Gastroenterology and Hepatology, Postbus 2040, 3000 CA Rotterdam, The Netherlands

Fax: +0031107030331

a.d.koch@erasmusmc.nl

\section{ABSTRACT}

Background and study aims Endoscopic retrograde cholangiopancreatography (ERCP) is considered one of the most technically demanding endoscopic procedures. Still, limited data are available on simulators in ERCP training.
Recently, the Boškoski-Costamagna ERCP Trainer was validated as a realistic training model by our study group. As an extension to this model, a novel synthetic papilla has been designed allowing to train biliary sphincterotomy. We aimed to determine the face validity of this synthetic papilla and its didactic value for training sphincterotomy.

Methods Expert participants, each with more a than 2500 ERCPs lifetime experience, were invited to perform a biliary sphincterotomy and fill out a questionnaire on the realism of the procedure and the didactic value.

Results A total of 40 ERCP experts were included, originating from 16 different countries. Experts' opinion on realism of performing a biliary sphincterotomy was rated with a median of 7 on a 10-point Likert scale, resemblance of the performed maneuvers 8 and tactile feedback 7. When asked if the cutting was perceived as realistic, experts rated a 6 and the cutting result was rated 8. The potential of the cutting papilla as a training tool for novices was rated 4 on a 4-point scale and there was a high agreement among the experts to include the papilla in the training of novices (rating 4).

Conclusion This is the first synthetic papilla available for training sphincterotomy on the Boškoski-Costamagna ERCP Trainer and it demonstrates good face validity. ERCP experts highly agree on its didactic value and added value in the training curriculum of novice endoscopists.

\section{Introduction}

Endoscopic retrograde cholangiopancreatography (ERCP) is one of the most technically demanding and high-risk procedures in gastrointestinal endoscopy. Traditionally, trainees have learned endoscopy by hands-on training in clinical setting on real patients under the supervision of an experienced endoscopist. In recent decades, simulators have assumed an important role in creating sufficient hands-on training without compromising patient safety and creating a safe learning environment for trainees [1]. Despite the fact that ERCP seems to be an ideal platform for simulation-based training, limited data are available on simulators in training ERCP. Recently, our study group has validated a novel mechanical ERCP simulator, the
Boškoski-Costamagna ERCP Trainer [2]. This simulator allows novice trainees to practice cannulation, stent placement in both common bile duct (CBD) and pancreatic duct (PD), and CBD stone extraction. To date, this simulator has not been equipped with a papilla that could be used to train endoscopic sphincterotomy.

Endoscopic sphincterotomy was first reported in 1974 and is one of the key therapeutic interventions during ERCP [3]. At the same time, it is also considered one of the riskier parts of the procedure. This is because of its technical difficulty and the associated risk of bleeding, perforation, and post-ERCP pancreatitis $[4,5]$. These complications are most often a result of an incorrectly performed sphincterotomy and frequently associated with trainees or inexperienced endoscopists [5]. First ex- 
perience in sphincterotomy is usually acquired through practice during live cases in an "on-the-job" setting. It goes without saying that, for both trainees and trainers alike - let alone the patient - this is not the ideal situation in which to do training on such a complex procedure. Ideally, such training requires a simulated setting that resembles the real thing as close as possible without jeopardizing patient safety and preferably with the ability to break down the procedure in steps that can be repeated as many times as necessary in order to gain proficiency. Currently, only a handful of training models are available for training ERCP and not every model is equipped for using in training on sphincterotomy. Available training models include in vivo and ex vivo training simulators, mechanical simulators, and virtual reality simulators [6-12]. Each training model has its own prominent features, but all the devices have important limitations, including ethical and practical concerns, differences in anatomy of the papilla, and lack of realism and tactile feedback. Up until now, no optimal training model for training sphincterotomy has been designed. A novel synthetic papilla has recently been developed and produced by Cook Medical (Cook Medical, Limerick, Ireland) for the Boškoski-Costamagna ERCP Trainer for use in training on sphincterotomy. This is a single-use synthetic papilla that can be easily replaced for repeated attempts at sphincterotomy. Validity assessment of a simulator can be performed on various levels. One of the most commonly used forms of validation is face validity. Face validity is evaluated by a defined group of subjects who are asked to judge the degree of resemblance between a training model and the real activity. The aim of this study was to evaluate the extent to which the novel papilla simulates real-life endoscopic biliary sphincterotomy, and to assess the didactic value or potential of the papilla for sphincterotomy training.

\section{Materials and methods}

\section{Synthetic papilla}

The synthetic papilla is a newly developed insertable component in the previously described mechanical ERCP simulator, the Boškoski-Costamagna ERCP Trainer (Cook Medical, Limerick, Ireland) [2]. It can be used to perform sphincterotomy. This novel synthetic papilla is made out of rubber and metal filaments and can be easily manually inserted into the BoškoskiCostamagna ERCP Trainer ( $\mathbf{F i g . 1}$ and $>$ Fig. 2). It is a singleuse disposable papilla, with a specific alloy allowing for electrical conduction and cutting of the material with all commercially available sphincterotomes and needle knives.

\section{Participants}

We included participants with broad experience in ERCP based on lifetime endoscopic experience. Expressing experience levels in ERCP remains debatable, and currently there is no consensus in the literature. In our previous study [2] we attempted to define four groups of participants according to the most reported numbers in the literature [13-15]. We set the bar for defining an individual as an ERCP expert at 2500 ERCPs to ensure that participants had an irrefutable reputation and broad experience in performing sphincterotomy.
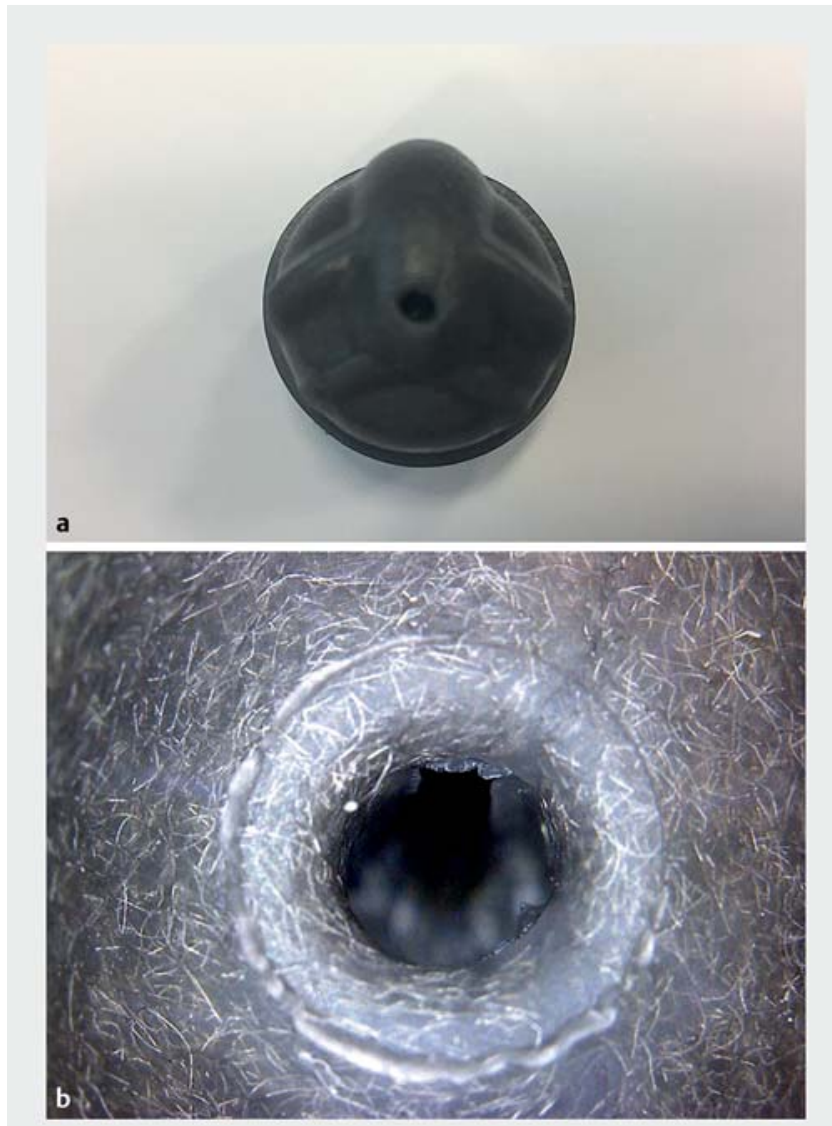

- Fig. 1 The synthetic papilla. a Front view of the papilla. b Closeup of the papilla, demonstrating the rubber with incorporated metal filaments.

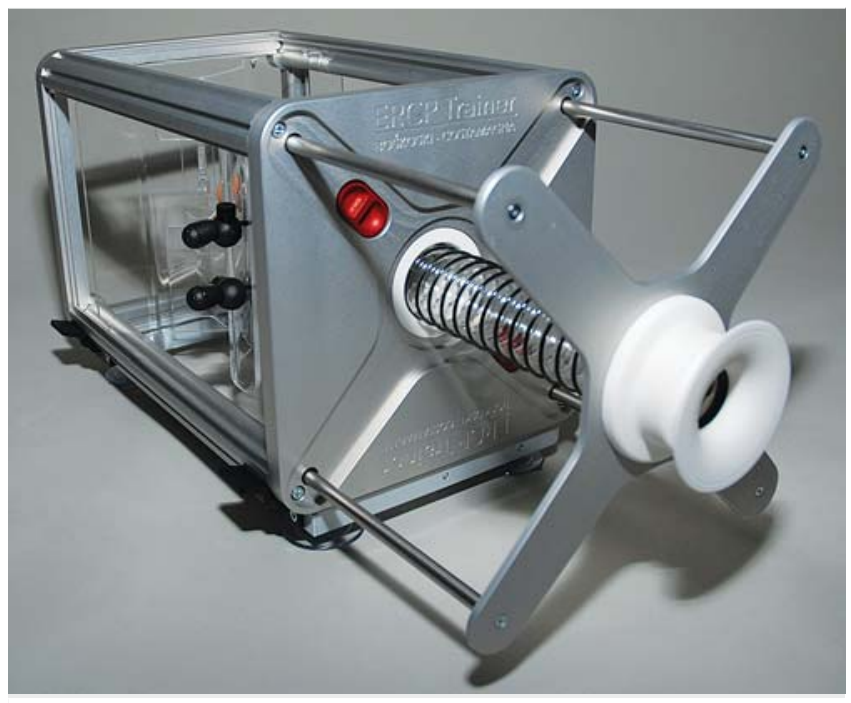

- Fig. 2 The Boškoski-Costamagna ERCP Trainer. (Source: Cook Medical, Bloomington, Indiana) 


\section{Sphincterotomy simulation setting}

All participants performed a simulator session during the course of the UEG Week in 2016 in Vienna, Austria. Experts were asked to perform a single biliary sphincterotomy and to fill out a questionnaire on demographics, medical experience, and endoscopy experience, including the numbers of ERCP procedures performed annually and estimated lifetime numbers. We asked participants to perform a sphincterotomy using a standard sphincterotome (Omnitome, Cook Medical, Limerick, Ireland) ( $\triangleright$ Video 1). Following this assignment, they were asked to rate their appreciation of the realism of the cutting papilla. Appreciation was expressed on a 10-point Likert scale [16], varying from very unrealistic (1) to very realistic (10). Questions were asked about the realism of performing sphincterotomy, anatomical representation, difficulty, the actual cutting, and the achieved cutting result. Furthermore, experts were asked to evaluate the didactic value of the cutting papilla on a 4-point Likert scale, varying from strongly disagree (1) to strongly agree (4).

\section{Data analysis}

Statistical analyses were performed using IMB SPSS Statistics, Version 24.0 (IBM Corporation, Armonk, New York, United States). Descriptive statistics were used for all measures. Data are presented as median and interquartile range. Inter-rater agreement and reliability were evaluated by computing the intraclass correlation coefficient (ICC) in a two-way mixed model.

\section{Results}

\section{Participants}

In total, 40 ERCP experts participated in this study originating from 16 different countries worldwide. Of the participants, 37 were gastroenterologists and the remaining three participants (7.5\%) were surgeons. All participants were male, with a mean age of 49.6 years (standard deviation 9). Mean number of years in practice as an endoscopist was 20.9 (interquartile

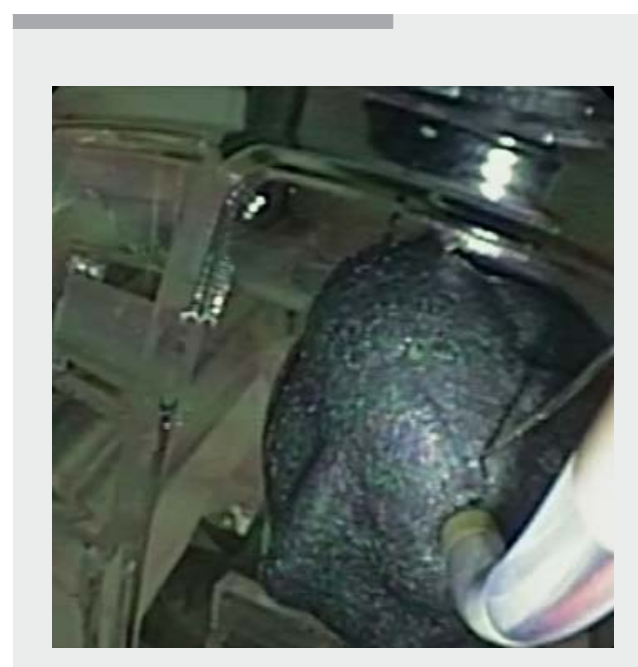

Video 1 Training sphincterotomy on the synthetic papilla. range 11). All participants completed the assignment and filled out the questionnaire. Baseline characteristics can be found in $>$ Table 1.

\section{Face validity}

The experts rated the anatomical representation of the synthetic papilla 8 on a 10-point Likert scale. Adequacy to position the endoscope in front of the papilla scored 9. $>$ Table 2 shows the experts' ratings on the papilla and its performance. The realism of performing a biliary sphincterotomy scored 7 on a 10point Likert scale. Resemblance of maneuvers compared to real life scored 8 by the experts and the associated tactile feedback scored 7 on a 10-point scale. The experts scored the cutting as realistic with a 6 and the cutting result was rated 8 on a 10 -point scale. The Intraclass Correlation Coefficient demonstrated strong agreement between the experts (ICC $=0.917)$, with a $95 \% \mathrm{Cl}$ of 0.796 to 0.983 .

\section{Didactic/training value}

Experts rated the papilla as a useful tool in basic training of novice endoscopists (4 on a 4-point scale; interquartile range [IQR] 0) and they unanimously agreed that the synthetic papilla should be incorporated in a training curriculum (4 on a 4-point scale; IQR 0). The experts' opinion was that the expertise gained with the synthetic papilla is transferrable into the clinical setting (4 on a 4-point scale; IQR 1). The role of the synthetic papilla in training more experienced ERCPist was rated to be limited according to the experts (rated 2 on a 4-point scale; IQR 3 ). The ICC for the average of the 40 experts was 0.983 with a $95 \% \mathrm{Cl}$ of 0.951 to 0.998 , indicating an excellent level of interrater agreement and reliability.

\section{Discussion}

Performing a sphincterotomy is one of the key elements in ERCP and is considered a challenging and high-risk procedure. Yet what that training of endoscopists should look like and how ample it should be to ensure that sphincterotomies are performed safely is a much-debated topic. The most obvious reason why current training practice is considered suboptimal is lack of a suitable, representative, and safe simulator training environment. Here we report the first results with and validation of a synthetic papilla that can be used for training sphincterotomy in the Boškoski-Costamagna ERCP Trainer. Data from the current study demonstrate good face validity and ERCP experts from all over the world highly agree on the didactic value and added value of this papilla in the training curriculum for novice endoscopists.

Recently, we presented the Boškoski-Costamagna ERCP Trainer, a mechanical simulator for training ERCP [2]. Experts agreed on the didactic strength of the simulator and the added value of this simulator in the training curriculum for novice endoscopists. An important limitation of this model was inability to train on sphincterotomy. Currently, most endoscopists are being trained in sphincterotomy - including their first attempts with the procedure - on real patients. This is not a desirable situation because there is little to no room for mistakes, 
- Table 1 Expert baseline characteristics.

\begin{tabular}{|l|c|}
\hline Characteristic & \\
\hline Male, $n(\%)$ & $40(100 \%)$ \\
\hline Age, years ${ }^{1}$ & $49.6(9)$ \\
\hline Number of countries & 16 \\
\hline Profession, $n(\%)$ & $37(92.5)$ \\
\hline - Gastroenterologist & $3(7.5)$ \\
\hline - Surgeon & $20.9(11)$ \\
\hline Endoscopic experience, years ${ }^{2}$ & \\
\hline $\begin{array}{l}\text { 1 Data are expressed as mean and standard deviation. } \\
{ }^{2} \text { Data are expressed as median and interquartile range. }\end{array}$ \\
\hline
\end{tabular}

potentially putting patients at risk. Furthermore, clinical training opportunities are limited by the available patients that require a papillotomy and the time and availability of an experienced endoscopist to train the novice ERCPist appropriately. The optimal learning environment is a setting where the procedure can be repeatedly simulated step by step in the most realistic way possible with room for mistakes, thus enabling trainees to train inexhaustibly and gain technical skills and confidence before being exposed to such a procedure in a real-life patient.

Despite the potential of currently available training models [6-12], all these simulators have certain limitations and an optimal model for training sphincterotomy has not been developed yet. In terms of realism, biosimulation models and live porcine models are superior $[8,17]$. Live anesthetized pig models have been shown to be adaptable to all procedural aspects of ERCP, including sphincterotomy. In a study by Sedlack et al. 48 participants followed a 2-day training course and showed an increase in confidence scores, especially in complex procedures such as needle-knife pre-cut sphincterotomy [18]. The Erlangen ERCP model [7], an ex vivo tissue model, has been scored as one of the most realistic and useful available training models [17]. Major drawbacks of both in vivo and ex vivo tissue models include important differences between human and porcine anatomy (e.g. location of papilla) and, importantly for training purpose of sphincterotomy, the papilla of the live porcine model can only be used once. The complete set-up requires a lot of preparation and is therefore probably only used in workshops and not in daily training. In addition, costs and organizational difficulties due to the ethical considerations make this type of simulator setup difficult to incorporate in a training curriculum. Known mechanical trainers for training ERCP include the ERCP Mechanical Simulator (EMS) and X-Vision ERCP Training System $[10,11]$. Training sphincterotomy on the X-Vision ERCP Training System [11] was carried out using an organic papilla. No details have been provided on the biomaterials that were used. The EMS [10] consists of a disposable papilla constructed of foam, held in place by electrical contacts with conducting gel providing electrical conductivity. Studies validating the use of the EMS for training sphincterotomy are lacking.
- Table2 Expert opinion on novel synthetic papilla.

\begin{tabular}{|l|l|}
\hline & $\begin{array}{l}\text { Expert opinion } \\
\mathbf{N}=\mathbf{4 0}\end{array}$ \\
\hline Resemblance to the real papilla & $8(4)$ \\
\hline Position in front of the papilla & $9(1)$ \\
\hline Realism of performing sphincterotomy & $7(1)$ \\
\hline Making the exact maneuvers as in real life & $8(1)$ \\
\hline Realism of haptic feedback & $7(3)$ \\
\hline Cutting is perceived as expected & $6(3)$ \\
\hline Cutting result & $8(1)$ \\
\hline Data are expressed as median and interquartile range & \\
\hline
\end{tabular}

Computer modules for training endoscopic sphincterotomy are available on the Simbionix GI Mentor, with the possibility to train on possible complications as well (e.g. bleeding, perforation). However, the model has received low scores for realism because of a major lack of tactile feedback and control of handling real equipment [13].

All experts in our study agreed that the novel synthetic papilla mimics the human papilla well for training biliary sphincterotomy. They were satisfied with the realism of performing a sphincterotomy, mainly with the opportunity to practice the exact maneuvers needed to correctly perform the procedure. Tactile feedback fromf the synthetic papilla was evaluated positively, despite the fact that the papilla is constructed from rubber. This probably also explains why the cutting effect was rated only 6 as it is not the same as cutting living tissue. The obvious advantage of the synthetic papilla is that it is easily replaced by a new one after it has been cut, thereby offering the possibility of repeatedly training on the procedure. Importantly, its use is not impeded by ethical concerns, given the mechanical origin of the simulator and papilla. The expert opinion on the possibility of using the synthetic papilla as a training tool was considered of great value in our study and all agreed that the synthetic papilla is a useful tool in training novice endoscopists to perform sphincterotomy. Experts also agreed that the expertise gained on the papilla should be directly transferrable to a clinical curriculum.

Despite the enthusiasm of experts for the synthetic papilla as a training tool for biliary sphincterotomy, some limitations remain, including absence of simulation of potential complications such as bleeding and perforation. Also, due to the mechanical nature of the simulator, there is no peristalsis and therefore, endoscopic control and positioning still differs from the real-life situation.

Notwithstanding these limitations, we believe that this novel synthetic papilla offers added value over currently available simulator options for training sphincterotomy. It has the advantage that a real endoscope with real accessories are used in a validated mechanical ERCP trainer model, providing novice endoscopists with a safe opportunity to learn how to execute the necessary movements and actions under the supervision 
of an experienced endoscopists in order to perform a real sphincterotomy plus the ability to train on the various steps in the procedure as many times as desired.

\section{Conclusion}

This novel synthetic papilla is a validated tool for training biliary sphincterotomy on the Boškoski-Costamagna ERCP Trainer. We have demonstrated good face validity, meaning that this papilla can be used to perform sphincterotomies that resemble the real-life situation. ERCP experts highly agree on the didactic value and added value of this papilla in the training curriculum for novice endoscopists.

\section{Acknowledgements}

The authors thank the participants for the opportunity to evaluate the novel synthetic papilla.

\section{Competing interests}

Dr. Koch reports receiving personal fees from Cook Medical. Dr. Bruno reports receiving grants and personal fees from Boston Scientific, Cook Medical, and Pentax Medical; and personal fees from $3 \mathrm{M}$ and GastroUpdate, outside the submitted work.

\section{References}

[1] van der Wiel SE, Kuttner Magalhaes R, Rocha Goncalves CR et al. Simulator training in gastrointestinal endoscopy - From basic training to advanced endoscopic procedures. Best Pract Res Clin Gastroenterol 2016; 30: $375-387$

[2] van der Wiel SE, Koch AD, Bruno MJ. Face and construct validity of a novel mechanical ERCP simulator. Endosc Int Open 2018; 6: E758 E765

[3] Kawai K, Akasaka Y, Murakami K et al. Endoscopic sphincterotomy of the ampulla of Vater. Gastrointest Endosc 1974; 20: 148-151
[4] Cotton PB, Lehman G, Vennes J et al. Endoscopic sphincterotomy complications and their management: an attempt at consensus. Gastrointest Endosc 1991; 37: $383-393$

[5] Freeman ML, Nelson DB, Sherman $S$ et al. Complications of endoscopic biliary sphincterotomy. N Engl J Med 1996; 335: 909-918

[6] Frimberger E, von Delius S, Rosch T et al. A novel and practicable ERCP training system with simulated fluoroscopy. Endoscopy 2008; 40: $517-520$

[7] Neumann M, Mayer G, Ell C et al. The Erlangen Endo-Trainer: life-like simulation for diagnostic and interventional endoscopic retrograde cholangiography. Endoscopy 2000; 32: $906-910$

[8] Matthes K, Cohen J. The Neo-Papilla: a new modification of porcine ex vivo simulators for ERCP training (with videos). Gastrointest Endosc 2006; 64: 570 - 576

[9] Bar-Meir S. Simbionix simulator. Gastrointest Endosc Clin N Am 2006; 16: $471-478$, vii

[10] Leung JW, Lee JG, Rojany M et al. Development of a novel ERCP mechanical simulator. Gastrointest Endosc 2007; 65: 1056 - 1062

[11] von Delius S, Thies P, Meining A et al. Validation of the X-Vision ERCP Training System and technical challenges during early training of sphincterotomy. Clin Gastroenterol Hepatol 2009; 7: 389-396

[12] Itoi T, Gotoda T, Baron TH et al. Creation of simulated papillae for endoscopic sphincterotomy and papillectomy training by using in vivo and ex vivo pig model. Gastrointestinal Endoscopy 2013; 77: $793-800$

[13] Bittner JGt, Mellinger JD, Imam T et al. Face and construct validity of a computer-based virtual reality simulator for ERCP. Gastrointest Endosc 2010; 71: $357-364$

[14] Sahakian AB, Laine L, Jamidar PA et al. Can a Computerized Simulator Assess Skill Level and Improvement in Performance of ERCP? Dig Dis Sci 2016; 61: $722-730$

[15] Arnold SH, Svendsen MB, Konge L et al. Three-dimensional motion tracking correlates with skill level in upper gastrointestinal endoscopy. Endoscopy 2015; 47: 825-828

[16] Lickert R. A technique for the measurement of attitudes. Arch Psychol 1932; $22: 140-155$

[17] Sedlack R, Petersen B, Binmoeller K et al. A direct comparison of ERCP teaching models. Gastrointest Endosc 2003; 57: 886-890

[18] Sedlack RE, Petersen BT, Kolars JC. The impact of a hands-on ERCP workshop on clinical practice. Gastrointest Endosc 2005; 61: 67 - 71 